\title{
Integral Categories and Calculus Categories*
}

\author{
Robin Cockett ${ }^{1}$ and Jean-Simon Lemay ${ }^{2}$ \\ 1 Dept. of Computer Science, University of Calgary, Calgary, AB, Canada \\ robin@ucalgary.ca \\ 2 Dept. of Mathematics and Statistics, University of Calgary, Calgary, AB, \\ Canada \\ jeansimon.lemay@ucalgary.ca
}

\begin{abstract}
Differential categories are now an established abstract setting for differentiation. The paper presents the parallel development for integration by axiomatizing an integral transformation, $\mathrm{s}_{A}: ! A \rightarrow ! A \otimes A$, in a symmetric monoidal category with a coalgebra modality. When integration is combined with differentiation, the two fundamental theorems of calculus are expected to hold (in a suitable sense): a differential category with integration which satisfies these two theorem is called a calculus category.

Modifying an approach to antiderivatives by T. Ehrhard, it is shown how examples of calculus categories arise as differential categories with antiderivatives in this new sense. Having antiderivatives amounts to demanding that a certain natural transformation, $\mathrm{K}: ! A \rightarrow ! A$, is invertible. We observe that a differential category having antiderivatives, in this sense, is always a calculus category and we provide examples of such categories.
\end{abstract}

1998 ACM Subject Classification F.3.3 Studies of Program Constructs, F.4.1 Mathematical Logic

Keywords and phrases Differential Categories, Integral Categories, Calculus Categories

Digital Object Identifier 10.4230/LIPIcs.CSL.2017.20

\section{Introduction}

The two fundamental theorems of calculus relate the two most important operations of calculus: differentiation and integration. The first theorem states that the derivative of the integral of a real function $f$ is the original function: $\frac{\mathrm{d}\left(\int_{a}^{t} f(x) \mathrm{d} x\right)}{\mathrm{d} t}(x)=f(x)$. While the second states that the integral of the derivative of a real function $f$ on a closed interval $[a, b]$ is equal to the difference of $f$ evaluated at the end points: $\int_{a}^{b} \frac{\mathrm{d} f(t)}{\mathrm{d} t}(x) \mathrm{d} t=f(b)-f(a)$. They are called "fundamental" theorems because they are absolutely fundamental to the development of classical calculus.

Since the turn of the $21^{\text {st }}$ century, there has been significant progress in the abstract understanding of differentiation with the study of differential categories. The abstract formulation of integration, on the other hand, has not received the same level of attention. Nonetheless, one might expect that, when suitably adjoined to the formulation of differentiation, a commensurate abstract form for integration should encompass these fundamental theorems. The purpose of this extended abstract is to explore the extent to which this expectation is realized.

* This work was partially supported by NSERC, Canada.

cc) (i) $\odot$ Robin Cockett and Jean-Simon Lemay;

licensed under Creative Commons License CC-BY

26th EACSL Annual Conference on Computer Science Logic (CSL 2017).

Editors: Valentin Goranko and Mads Dam; Article No. 20; pp. 20:1-20:17

Leibniz International Proceedings in Informatics

LIPICS Schloss Dagstuhl - Leibniz-Zentrum für Informatik, Dagstuhl Publishing, Germany 
In the early 2000's, T. Ehrhard and L. Regnier introduced the differential $\lambda$-calculus [12] and differential proof nets [13], which formalized differentiation in linear logic. A few years later, R. Blute, R. Cockett and R. Seely introduced differential categories [7], which were the appropriate categorical structure for modelling Ehrhard and Regnier's differential linear logic. Differential categories now have a rich literature of their own $[4,3,2,6,14,10,9]$ and there are many examples which have been extensively studied $[7,11,5]$. However, as mentioned above, little attention has been given to abstracting integration.

In 2014, T. Ehrhard observed that in certain $*$-autonomous categories which had the appropriate structure to be a differential category, it was possible with one additional assumption to produce antiderivatives [11]. The additional assumption was that a certain natural transformation - which he called $\mathbf{J}$ - constructed from the deriving transformation was a natural isomorphism. With this assumption, Ehrhard constructed an integral transformation with an inverse behaviour to the deriving transformation, in the sense that he gave necessary and sufficient conditions for a map to satisfy the first fundamental theorem of calculus - by proving Poincaré's Lemma. Furthermore, when the deriving transformation satisfied an extra - non-equational - condition, which he called the "Taylor Property", he then showed that every differentiable function satisfied the second fundamental theorem of calculus.

While much of the inspiration for our approach to integration derives from these observations, Ehrhard made no attempt to axiomatize integration separately from differentiation. Here we introduce (tensor) integral category as a notion which stands on its own (i.e. in the absence of differentiation). The inspiration for this independent axiomatization of integral categories comes from the much older notion of a Rota-Baxter algebra [1, 20, 15], the classical algebraic abstraction of integration. Briefly, for a commutative ring $R$ and $\lambda \in R$, a Rota-Baxter algebra of weight $\lambda$ is an $R$-algebra $A$ with an $R$-linear morphism $P: A \rightarrow A$ which satisfies the Rota-Baxter rule: $P(a) P(b)=P(a P(b))+P(P(a) b)+\lambda P(a b)$ for all $a, b \in A$. The map $P$ is called a Rota-Baxter operator of weight $\lambda$. A particular example of a Rota-Baxter algebra of weight zero is the $\mathbb{R}$-algebra of real continuous functions $\operatorname{Cont}(\mathbb{R})$, where the Rota-Baxter operator $P: \operatorname{Cont}(\mathbb{R}) \rightarrow \operatorname{Cont}(\mathbb{R})$ is defined as the integral of the function centred at zero: $P(f)(x)=\int_{0}^{x} f(t) \mathrm{d} t$. The Rota-Baxter rule for this example is the expression of the integration by parts rule without the use of derivatives: $\int_{0}^{x} f(t) \mathrm{d} t \cdot \int_{0}^{x} g(t) \mathrm{d} t=\int_{0}^{x} f(t) \cdot\left(\int_{0}^{t} g(u) \mathrm{d} u\right) \mathrm{d} t+\int_{0}^{x}\left(\int_{0}^{t} f(u) \mathrm{d} u\right) \cdot g(t) \mathrm{d} t$ (see [15] for more details). This motivates the Rota-Baxter rule as an axiom of integration.

When differentiation and integration are combined into what we call here a calculus category, we demand that the two fundamental theorems hold. The second fundamental theorem is assumed to hold verbatim. However, the first fundamental theorem, as above, has to be interpreted as being on maps - rather than objects - and, under this interpretation, becomes the Poincaré property, a conditional property which provides necessary and sufficient conditions for a map to be the differential of its integral. The name of the condition comes from the Poincaré Lemma from cohomology [22] and differential topology [8], which states an analogous result of giving criteria for a map to be an antiderivative.

To obtain the notion of integration as an antiderivative, we insist that a slightly different natural transformation, which we call $\mathrm{K}$, should be invertible. We show this is equivalent to requiring both that Ehrhard's transformation J is invertible and that the "Taylor Property" which Ehrhard had suggested was desirable - holds. This improvement is easily underestimated: the "Taylor Property" is a conditional requirement, replacing a conditional requirement by a purely equational requirement is always, mathematically, a significant step. Demanding that $\mathrm{K}$ is invertible not only produces an integral transformation, but also secures the first and second fundamental theorem of calculus. Inverting only Ehrhard's transformation, J, 
does not by itself even produce an integral transformation; the "Taylor Property" is required, in addition to the invertibility of $\mathrm{J}$, to secure an integral transformation. The fact that, when $\mathrm{K}$ is invertible, $\mathrm{J}$ is invertible is useful particularly in the proof of the Poincaré's lemma. Thus, it is important to observe that, the antiderivative produced by the inverse of $\mathrm{K}$ is precisely the same as the antiderivative produced by the inverse of $\mathrm{J}$-when $\mathrm{K}$ is already invertible.

Finally, the notion of a differential category with anti-derivatives, given by requiring $\mathrm{K}$ to be invertible, provides a plentiful supply of calculus categories as we explain.

Before beginning, we should mention the conventions that we use in the paper. First off, we will use diagrammatic order for composition. Explicitly, this means that the composite map $f g$ is the map which first does $f$ then $g$. Secondly, to simplify working in symmetric monoidal categories, we will allow ourselves to work in strict symmetric monoidal categories and so will generally suppress the associator and unitor isomorphisms. For a symmetric monoidal category we will use $\otimes$ for the tensor product, $I$ for the monoidal unit, and $\sigma: A \otimes B \rightarrow B \otimes A$ for the symmetry isomorphism.

Full detailed proofs of all the results in this extended abstract can be found in the second author's masters thesis [18].

\section{Coalgebra Modalities}

Tensor integral and differential categories are structures over additive symmetric monoidal categories with a coalgebra modality. We begin by recalling the components of this structure starting with the notion of an additive category. Here we mean "additive" in the sense of being commutative monoid enriched. Thus, we do not assume negatives nor do we assume biproducts (this differs from the usage in [19] for example). This allows many important examples such as the category of sets and relation or the category of modules of a commutative rig $^{1}$.

- Definition 1. An additive category is a commutative monoid enriched category, that is a category in which each hom-set is a commutative monoid - with addition operation + and zero 0 - and in which composition preserves addition that is:

[Add.1] $k(f+g)=k f+k g$ and $0 f=0$;

[Add.2] $(f+g) h=f h+g h$ and $f 0=0$.

An additive symmetric monoidal category is an additive category with a tensor product which is compatible with the additive structure in the sense that:

$\left[\mathbf{A d d}_{\otimes} \cdot \mathbf{1}\right](f+g) \otimes h=f \otimes h+g \otimes h$ and $0 \otimes h=0$;

[Add $\otimes .2] k \otimes(f+g)=k \otimes f+k \otimes g$ and $h \otimes 0=0$.

In any additive category their is a notion of "scalar multiplication" of maps by the natural numbers $\mathbb{N}$. The scalar multiplication of a map $f: A \rightarrow B$ by $n \in \mathbb{N}$, is the map $n \cdot f: A \rightarrow B$ defined by summing $n$ copies of $f$ together. If $n=0$, then $0 \cdot f$ is simply the zero map from $A$ to $B$. Furthermore, for additive symmetric monoidal categories, one then has that $(n \cdot f) \otimes g=n \cdot(f \otimes g)=f \otimes(n \cdot g)$.

- Definition 2. A coalgebra modality $[7,3]$ on a symmetric monoidal category is a quintuple $(!, \delta, \varepsilon, \Delta, e)$ consisting of a comonad $(!, \delta, \varepsilon)$, a natural transformation $\Delta$ with

\footnotetext{
1 Rigs are also known as a semirings: they are rings without negatives.
} 
components $\Delta_{A}: ! A \rightarrow ! A \otimes ! A$, and a natural transformation $e$ with components $e_{A}: ! A \rightarrow I$ such that for each object $A$ :

(i) $\left(! A, \Delta_{A}, e_{A}\right)$ is a cocommutative comonoid, that is, the following diagrams commute:
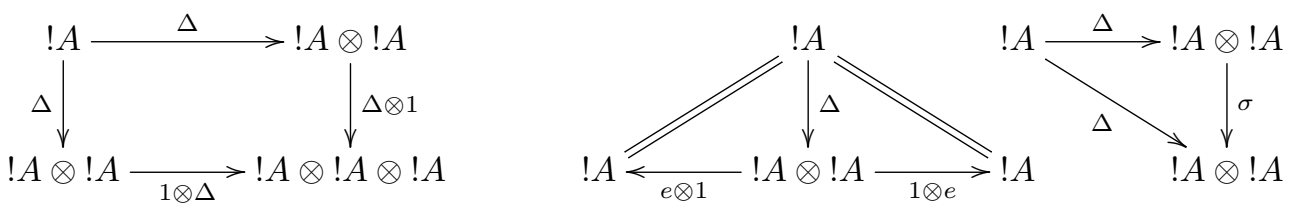

(ii) $\delta_{A}$ preserves the comultiplication, that is, the following diagram commutes:

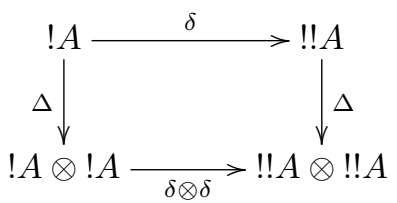

When combined with the additive structure, this ensures that $! A$ is a coalgebra in the classical algebraic sense. Furthermore, one can prove that $\delta e=e$ so $\delta$ is actually a comonoid homomorphism.

The coKleisli maps for the comonad are important: these maps are of the form $f: ! A \rightarrow B$ : amongst these are the linear maps $\varepsilon g: ! A \rightarrow B$ where $g: A \rightarrow B$.

Note that we do not assume that the coalgebra modality, !, is a monoidal functor: to do so would put us in the realm of Seely categories $[3,14]$ which is more than we require for this basic theory.

\section{Integral Categories}

Integral categories are the integral analogue of differential categories, thus, the main ingredient of an integral category is an integral transformation, $\mathrm{s}_{A}: ! A \rightarrow ! A \otimes A$, a natural transformation opposite in orientation to a deriving transformation which must satisfy just three equations:

- Definition 3. An additive symmetric monoidal category with a coalgebra modality is an integral category if there is a natural transformation $\mathrm{s}_{A}: ! A \rightarrow ! A \otimes A$, called the integral transformation, satisfying the following equations:

[s.1] Constants Rule: $\mathrm{s}(e \otimes 1)=\varepsilon$

[s.2] Rota-Baxter Rule: $\Delta(\mathrm{s} \otimes \mathrm{s})=\mathrm{s}(\Delta \otimes 1)(\mathrm{s} \otimes 1 \otimes 1)+\mathrm{s}(\Delta \otimes 1)(1 \otimes \sigma)(1 \otimes 1 \otimes \mathrm{s})$

[s.3] Interchange Rule: $\mathbf{s}(\mathbf{s} \otimes 1)=\mathbf{s}(\mathbf{s} \otimes 1)(1 \otimes \sigma)$

The integral of a map $f: ! A \otimes A \rightarrow B$ is defined as the composition $\mathrm{S}[f]:=\mathrm{s}_{A} f: ! A \rightarrow B$. This should be thought of as the classical integral of $f$ evaluated from 0 to $x$ as a function of $x: \mathrm{S}[f](x):=\int_{0}^{x} f(t) \mathrm{d} t$. To interpret this as $\mathrm{S}[f]$ one must regard $f$ as being a function of two variables $t$ and $\mathrm{d} t$, which is linear in $\mathrm{d} t$. Classically, $f$ is regarded as a function of one (one dimensional) variable, $t$, and to obtain the interpretation as a function of two arguments one simply multiplies by the variable $\mathrm{d} t$. This allows a simple interpretation of the integral notation for one dimensional functions: it leaves open the interpretation for multidimensional functions - an issue to which we shall return.

The additive structure of the category ensures the integral of a sum of maps is equal to the sum of the integral of each map, that is, $\mathrm{S}[f+g]=\mathrm{S}[f]+\mathrm{S}[g]$ and $\mathrm{S}[0]=0$. The first axiom [s.1] states that the integral of a constant map is a linear map (in the sense discussed above). The second axiom [s.2] is the Rota-Baxter rule [15], which is an expression 
of integration by parts using only integrals. In classical calculus notation, the Rota-Baxter rule is expressed as: $\int_{0}^{x} f(t) \mathrm{d} t \cdot \int_{0}^{x} g(t) \mathrm{d} t=\int_{0}^{x} f(t) \cdot\left(\int_{0}^{t} g(u) \mathrm{d} u\right) \mathrm{d} t+\int_{0}^{x}\left(\int_{0}^{t} f(u) \mathrm{d} u\right) \cdot g(t) \mathrm{d} t$. The third axiom [s.3] ensures the independence of the order of integration - the interchange law - that is integrating with respect to $u$ then $t$ is the same as integrating with respect to $t$ then $u$. It may be tempting to think this is related to Fubini's theorem. In fact, it is not closely related at all: we discuss this at the end of this section. [s.3] can be expressed in classical notation as: $\int_{0}^{x}\left(\int_{0}^{t} f(u) \mathrm{d} u \mathrm{~d} t\right)=\int_{0}^{x}\left(\int_{0}^{u} f(t) \mathrm{d} t \mathrm{~d} u\right)$.

Just like differential categories, integral categories have a graphical calculus (see [21] for an introduction to the graphical calculus in monoidal categories and its variations). We represent the integral transformation in string diagrams as follows (which should be read from top to bottom):<smiles>[Al]CC([Al])C[Al]</smiles>

The integral axioms [s.1], [s.2] and [s.3] are then represented in the graphical calculus as follows (we omit writing the objects at the end of the wires).

[s.1] Constants Rule:<smiles>CCOC</smiles>

\section{[s.2] Rota-Baxter Rule:}

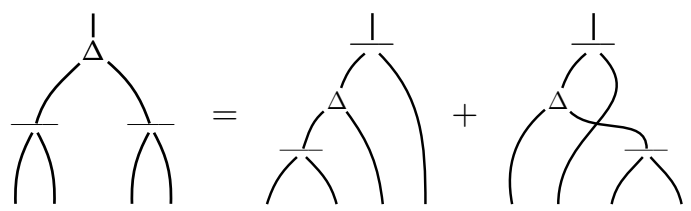

[s.3] Interchange Rule:<smiles>CC1(C)C2CCC1(C)C2(C)C</smiles>

With the graphical calculus, we are now in a position to explore polynomial integration. Perhaps the first formula learnt in first year calculus is $\int_{0}^{x} x^{n} \mathrm{~d} x=\frac{1}{n+1} x^{n+1}$. However this formula cannot be expressed in a general additive category - simply because there may not be fractions. That said, we will soon see that in every integral category there is a notion of scalar multiplication by positive rationals, that is, certain hom-sets are $\mathbb{Q} \geq 0$-modules, where $\mathbb{Q}_{\geq 0}$ is the rig of non-negative rationals. The integral of monomials identity can be re-express as the requirement that $(n+1) \int_{0}^{x} x^{n} \mathrm{~d} x=x^{n+1}$ and this identity does hold in any integral category! To express this identity in an integral category, we will need the $n$-fold comultiplication $\Delta_{n}: ! A \rightarrow ! A^{\otimes^{n}}$ which is defined as $\Delta_{n}=\Delta(\Delta \otimes 1)(\Delta \otimes 1 \otimes 1) \ldots\left(\Delta \otimes 1^{\otimes^{n-2}}\right)$. By convention we set $\Delta_{0}=e, \Delta_{1}=1$ and $\Delta_{2}=\Delta$. 
- Theorem 4. For every $n \in \mathbb{N}$, the integral transformation satisfies the polynomial identity: [s. Poly] $(n+1) \cdot \mathrm{s}\left(\Delta_{n} \otimes 1\right)\left(\varepsilon^{\otimes^{n}} \otimes 1\right)=\Delta_{n+1}\left(\varepsilon^{\otimes^{n+1}}\right)$<smiles>CO[Al](C)OC</smiles>

Proof. The beauty of this proof is that it uses every integral transformation axiom. The proof is much smoother using the graphical calculus, which is equivalent to proofs done algebraically as shown in [16]. We will prove the equality for the integral transformation by induction on $n$. For the base case of $n=0$, this equality holds directly by the constant rule [s.1]. Assume the induction hypothesis [s. Poly] holds for $n$, we now show it for $n+1$ :

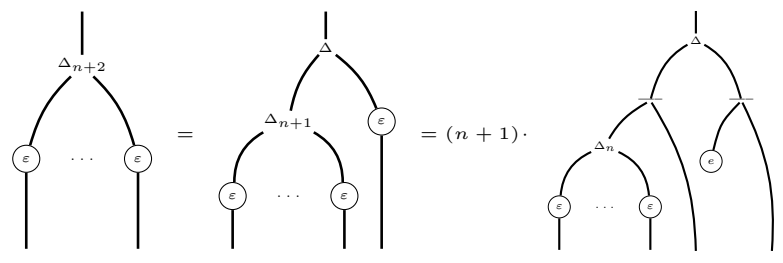

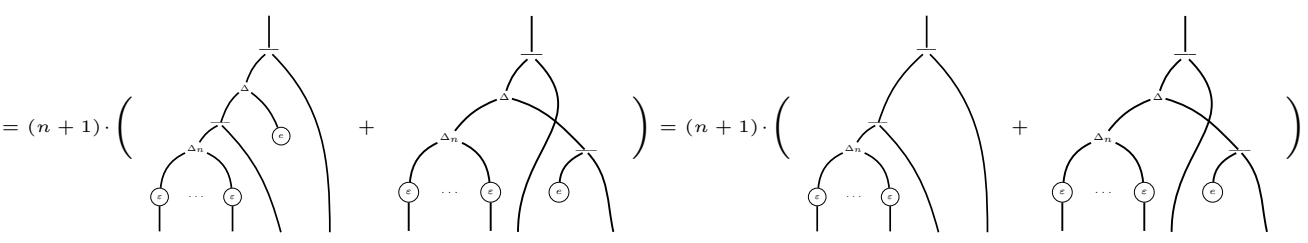<smiles>COC(C)[Al](C)OC</smiles><smiles>COC(C)[Al]C(C)[Al](C)OC</smiles><smiles>COC[C@@H]1C2CC3(C)CC1C(C)(C)C23</smiles><smiles>COC(=O)C(C)[Al](OC)OC</smiles><smiles>COC[As]CC(C)COC</smiles><smiles>CO[Al](OC)C(C)[18O]C</smiles>

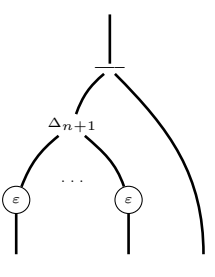

An important consequence of polynomial integration is that certain hom-sets are $\mathbb{Q}_{\geq 0^{-}}$ modules. In an additive category, for every object $A$ and for every natural number $n \in \mathbb{N}$, define the map $n_{A}: A \rightarrow A$ by summing $n$ copies of the identities: $n_{A}=n \cdot 1_{A}$. We will now prove that in any integral category that for every object $A$ and $n \geq 2$, the map $n_{!} A$ is invertible.

- Theorem 5. In an integral category, for every natural number $n \in \mathbb{N}, n \geq 2$, and every object $A \in \mathbb{X}$, the map $n_{! A}: ! A \rightarrow ! A$ is an isomorphism.

- Remark. Notice that the case $n=1$ is also true since the identity map is an isomorphism. 
Proof. We will simply define the inverse of $n_{!} A$. For each object $A$ and $n \geq 2$, define $n_{! A}^{-1}: ! A \rightarrow ! A$, as: $n_{! A}^{-1}=\delta_{A} \mathrm{~s} ! A\left(\Delta_{n-1} \otimes 1\right)\left(\varepsilon_{! A}^{\otimes^{n-1}} \otimes 1\right)\left(1 \otimes e^{\otimes^{n-1}}\right)$, written in the graphical calculus as:

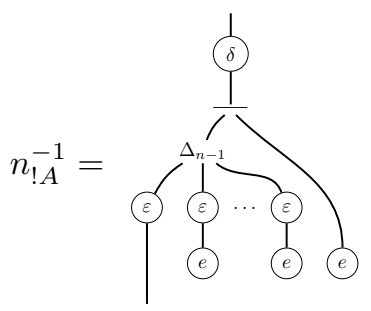

This implies, in an integral category, hom-sets with domain $! A$ are $\mathbb{Q} \geq 0$-modules. The scalar multiplication of a map $f: ! A \rightarrow B$ with a non-negative rational $\frac{p}{q} \in \mathbb{Q}_{\geq 0}$ is the map $\underline{p} \cdot f: ! A \rightarrow B$ defined as $\frac{p}{q} \cdot f=q_{! A}^{-1}(p \cdot f)$.

Finally, we discuss the interpretation of Fubini's theorem. The theorem requires that the coalgebra modality is monoidal and, thus, that there is a Seely isomorphism [2, 14]: $\chi: ! A \otimes ! B \rightarrow !(A \times B)$. Fubini's theorem concerns the double integration of a function of the form $f: !(A \times B) \otimes A \otimes B \rightarrow C$ whose type ensures it is bilinear in the second two occurrences of $A$ and $B$. Functions of this form can be integrated with respect to either $A$ or $B$, or both $A$ and $B$ : the latter, the double integral of $f$, is obtained as follows:

$$
!(A \times B) \stackrel{\chi^{-1}}{\longrightarrow} ! A \otimes ! B \stackrel{s \otimes s}{\longrightarrow} ! A \otimes A \otimes ! B \otimes B \stackrel{1 \otimes \sigma \otimes 1}{\longrightarrow} ! A \otimes ! B \otimes A \otimes B \stackrel{\chi \otimes 1 \otimes 1}{\longrightarrow} !(A \times B) \otimes A \otimes B \stackrel{f}{\longrightarrow} C
$$

Fubini's theorem asserts that the order of integration in this double integral does not matter. At this level of generality this order independence is an immediate consequence of the bifunctoriality of $\_\otimes_{\ldots}$.

\section{Calculus Categories}

In this section we wish to put integration together with differentiation and to discuss how they should interact. We start by briefly recalling the definition of a differential category [7] before introducing calculus categories whose structure is induced by the fundamental theorems of calculus.

- Definition 6. An additive symmetric monoidal category with a coalgebra modality is a differential category if the coalgebra modality comes equipped with a deriving transformation [7], that is, a natural transformation $\mathrm{d}$ with components $\mathrm{d}_{A}: ! A \otimes A \rightarrow ! A$, satisfying the following equations:

[d.1] Constant Rule: de $=0$

[d.2] Leibniz Rule: $\mathrm{d} \Delta=(\Delta \otimes 1)(1 \otimes \sigma)(\mathrm{d} \otimes 1)+(\Delta \otimes 1)(1 \otimes \mathrm{d})$

[d.3] Linear Rule: $\mathrm{d} \varepsilon=(e \otimes 1) \lambda$

[d.4] Chain Rule: $\mathrm{d} \delta=(\Delta \otimes 1)(\delta \otimes 1 \otimes 1)(1 \otimes \mathrm{d}) \mathrm{d}$

[d.5] Interchange Rule: $(\mathrm{d} \otimes 1) \mathrm{d}=(1 \otimes \sigma)(\mathrm{d} \otimes 1) \mathrm{d}$

The derivative of a map $f: ! A \rightarrow B$ is the composition $\mathrm{D}[f]:=\mathrm{d}_{A} f: ! A \otimes A \rightarrow B$. The first axiom, $[\mathbf{d . 1}]$, states that the derivative of a constant map is zero. The second axiom [d.2] is the Leibniz rule for differentiation - also called the product rule. The third axiom [d.3] says that the derivative of a linear map is a constant. The fourth axiom [d.4] is the chain rule. The last axiom [d.5] is the independence of differentiation or the interchange law, which naively states that differentiating with respect to $x$ then $y$ is the same as differentiation with respect to $y$ then $x$. It should be noted that [d.5] was not a requirement in [7] but 
was later added to the definition $[3,3]$ to ensure that the coKleisli category of a differential category was a Cartesian differential category.

As previously stated, differential categories have a graphical calculus. The deriving transformation is represented as follows:<smiles>[Al]C1CCCC1</smiles>

The string diagram representations of $[\mathbf{d . 1}]$ to $[\mathbf{d . 5}]$ are as follows:

[d.1] Constant Rule:<smiles>CCC(C)C</smiles>

[d.2] Leibniz Rule:<smiles>CCC(CC)C(CC)CC</smiles>

[d.3] Linear Rule:

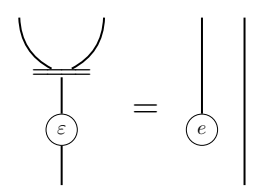

[d.4] Chain Rule:

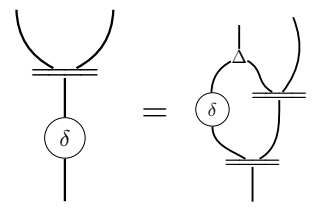

[d.5] Interchange Rule:

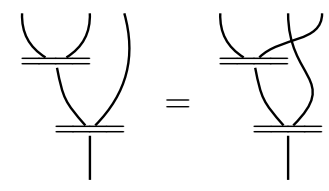

We are now ready to tackle the interaction between integration and differentiation. We start with the second fundamental theorem of calculus and return to discuss the first fundamental theorem of calculus:

- Definition 7. Let $\mathbb{X}$ be a differential category and an integral category with deriving transformation $\mathrm{d}$ and integrating transformation $\mathrm{s}$ on the same coalgebra modality $(!, \delta, \varepsilon, \Delta, e)$. 
(i) $d$ and $s$ are said to satisfy the Second Fundamental Theorem of Calculus if: sd $+! 0=1$, written in the graphical calculus as:

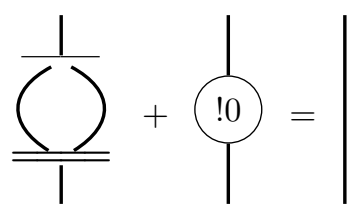

(ii) $d$ and $s$ are said to be compatible if: $d s d=d$, written in the graphical calculus as:

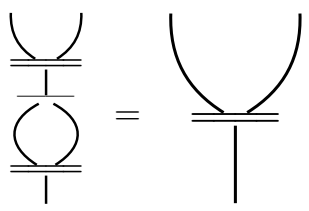

(iii) $\mathrm{d}$ is said to be Taylor if for every pair of maps $f, g: C \otimes ! A \rightarrow B$, such that

$$
(1 \otimes \mathrm{d}) f=(1 \otimes \mathrm{d}) g
$$

then $f+(1 \otimes !(0)) g=g+(1 \otimes !(0)) f$.

The first part of the definition expresses the second fundamental theorem of calculus. Compatibility is a weaker version of the second fundamental theorem. The Taylor property (see [11]) is the property that if two maps have the same derivative then they differ by constants.

- Theorem 8. For a deriving transformation $\mathrm{d}$ and an integral transformation $\mathrm{s}$ on the same coalgebra modality, the following are equivalent:

(i) d and s satisfy the Second Fundamental Theorem of Calculus;

(ii) $\mathrm{d}$ and $\mathrm{s}$ are compatible and $\mathrm{d}$ is Taylor.

Remark. This is an extension of Proposition 14 of [11] which proved (ii) $\Rightarrow$ (i) for Ehrhard's original integral using $\mathrm{J}^{-1}$, however the notion of compatibility was not identified although it was used in the proof.

Proof. (i) $\Rightarrow$ (ii): Suppose d and s satisfy the Second Fundamental Theorem of Calculus. For Taylor, suppose that $(1 \otimes \mathrm{d}) f=(1 \otimes \mathrm{d}) g$. Then we have the following equality:

$$
f+(1 \otimes ! 0) g=(1 \otimes \mathbf{s})(1 \otimes \mathbf{d}) f+(1 \otimes ! 0) f+(1 \otimes ! 0) g=(1 \otimes \mathbf{s})(1 \otimes \mathbf{d}) g+(1 \otimes ! 0) f+(1 \otimes ! 0) g=g+(1 \otimes ! 0) f
$$

For compatibility, by naturality, we have the following equality:

$$
\mathrm{d}=\mathrm{dsd}+\mathrm{d} !(0)=\mathrm{dsd}+(!(0) \otimes 0) \mathrm{d}=\mathrm{dsd}+0=\mathrm{dsd} .
$$

(i) $\Rightarrow$ (ii): Suppose $d$ and s are Compatible and $d$ is Taylor. Notice by Compatibility we have: $\mathrm{dsd}=\mathrm{d}$, and then by Taylor (where $f=\mathrm{sd}$ and $g=1$ ) we have the following equality: sd $+!(0)=1+!(0)$ sd. However, using naturality, we have:

$$
\mathrm{sd}+!(0)=1+!(0) \mathrm{sd}=1+\mathbf{s}(!(0) \otimes 0) \mathrm{d}=1+0=1 .
$$

The interpretation of the first fundamental theorem of calculus, unlike the second fundamental theorem, is as a property of a map: 
- Definition 9. A map $f: C \otimes ! A \otimes A \rightarrow B$ satisfies the First Fundamental Theorem (in the last two arguments) if $\left(1 \otimes\left(\mathrm{d}_{A} \mathrm{~s}_{A}\right)\right) f=f$, written in the graphical calculus as:<smiles>CCC(C)(C)C1CC2CCCCC21</smiles>

Thus, if $f$ satisfies the First Fundamental theorem, it may be viewed as the differential of a map - namely the differential of its integral. Clearly not all maps will satisfy the First Fundamental theorem calculus, a necessary condition is:

- Lemma 10. If a map, $f: C \otimes ! A \otimes A \rightarrow B$, satisfies the First Fundamental Theorem, then: $(1 \otimes 1 \otimes \sigma)(1 \otimes \mathrm{d} \otimes 1) f=(1 \otimes \mathrm{d} \otimes 1) f$.

Proof. As $(1 \otimes \mathrm{ds}) f=f$, the interchange rule for the deriving transformation [d.5] gives:

$$
(1 \otimes 1 \otimes \sigma)(1 \otimes \mathrm{d} \otimes 1) f=(1 \otimes 1 \otimes \sigma)(1 \otimes \mathbf{d} \otimes 1)(1 \otimes(\mathrm{ds})) f=(1 \otimes \mathrm{d} \otimes 1)(1 \otimes(\mathrm{ds})) f=(1 \otimes \mathrm{d} \otimes 1) f
$$

We shall use the converse of this lemma as an axiom and call it the Poincaré condition:

- Definition 11. A differential category with an integral transformation satisfies the Poincaré condition if any map $f: C \otimes ! A \otimes A \rightarrow B$ for which: $(1 \otimes 1 \otimes \sigma)(1 \otimes \mathbf{d} \otimes 1) f=(1 \otimes \mathbf{d} \otimes 1) f$, satisfies the First Fundamental Theorem - that is: $(1 \otimes \mathrm{ds}) f=f$.

The Poincaré condition and Lemma 10 imply the following equivalence:

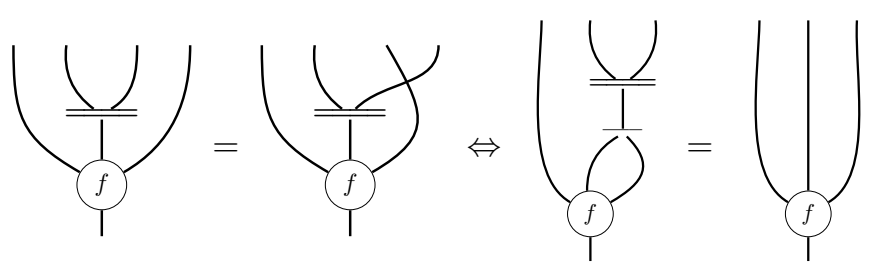

The Poincaré condition also implies compatibility of the deriving transformation and integrating transformation.

- Theorem 12. The integral and deriving transformation are compatible in any differential category with an integral transformation which satisfies the Poincaré condition.

Proof. By [d.5], the deriving transformation d satisfies the Poincaré pre-condition that $(1 \otimes \sigma)(\mathrm{d} \otimes 1) \mathrm{d}=(\mathrm{d} \otimes 1) \mathrm{d}$. Therefore, $\mathrm{d}$ satisfies the First fundamental theorem of Calculus, which is simply the statement of compatibility: $d s d=d$.

- Corollary 13. A deriving and integral transformation which satisfy the Poincaré condition such that d is Taylor, satisfies the Second Fundamental Theorem of Calculus.

This suggests the following basic definition:

- Definition 14. A calculus category is a differential category and an integral category on the same coalgebra modality such that the deriving transformation and the integral transformation satisfy the Second Fundamental Theorem of Calculus and the Poincaré condition. 


\section{$5 \quad$ Antiderivatives}

In this last section, we explore how one obtains a calculus category from a differential category with "antiderivatives". A differential category has "antiderivatives" when a certain natural transformation, $\mathrm{K}$ - which is present in all differential categories - is a natural isomorphism. This is a strengthening of Ehrhard's original idea in [11], which required a different natural transformation, $\mathrm{J}$, to be a natural isomorphism. Inverting $\mathrm{J}$ by itself does not appear to give even an integral category: to obtain an integral category and the second fundamental theorem of calculus Ehrhard also demanded the Taylor property. Inverting K, as we shall see, gets all these properties - and, thus, a calculus category - in one step.

In an additive symmetric monoidal category with a coalgebra modality, the coderiving transformation is the natural transformation $\mathrm{d}_{A}^{\circ}:=\Delta_{A}\left(1_{! A} \otimes \varepsilon_{A}\right): ! A \rightarrow ! A \otimes A$ (this called the "annihilation operator" in [14]). We represent the coderiving transformation as an upside down deriving transformation in the graphical calculus:

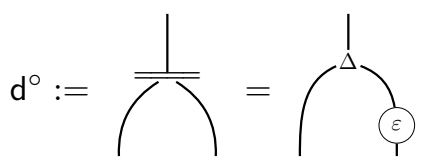

The coderiving transformation would probably be one's first attempt at constructing an integrating transformation in differential category. The following theorem indicates how close the coderiving transformation is to being an integrating transformation:

- Theorem 15. The coderiving transformation $\mathrm{d}^{\circ}$ satisfies the following properties:

[cd.1] $\mathrm{d}^{\circ}(e \otimes 1)=\varepsilon$

[cd.2] $\mathrm{d}^{\circ}(\varepsilon \otimes 1)=\Delta(\varepsilon \otimes \varepsilon)$

[cd.3] $\mathrm{d}^{\circ}(\Delta \otimes 1)=\Delta\left(1 \otimes \mathrm{d}^{\circ}\right)$

[cd.4] $\mathrm{d}^{\circ}(\Delta \otimes 1)(1 \otimes \sigma)=\Delta\left(\mathrm{d}^{\circ} \otimes 1\right)$

[cd.5] $2 \cdot \Delta\left(\mathrm{d}^{\circ} \otimes \mathrm{d}^{\circ}\right)=\mathrm{d}^{\circ}(\Delta \otimes 1)\left(\mathrm{d}^{\circ} \otimes 1 \otimes 1\right)+\mathrm{d}^{\circ}(\Delta \otimes 1)(1 \otimes \sigma)\left(1 \otimes 1 \otimes \mathrm{d}^{\circ}\right)$

[cd.6] $\mathrm{d}^{\circ}(\delta \otimes 1)=\delta \mathrm{d}^{\circ}(1 \otimes \varepsilon)$

[cd.7] $d^{\circ}\left(d^{\circ} \otimes 1\right)=d^{\circ}\left(d^{\circ} \otimes 1\right)(1 \otimes \sigma)$

Notice in particular [cd.1], [cd.5] and [cd.7]. If we let $s=\mathrm{d}^{\circ}$, then [cd.1] and [cd.7] are precisely the same as [s.1] and [s.3]. However, the coderiving transformation fails to satisfy [s.2], the Rota-Baxter rule, since [cd.5] has an extra factor of 2. Of course, if the differential category is in fact enriched over idempotent commutative monoids, so that $1+1=1$, the coderiving transformation would be an integral transformation: this happens, for example, in the category of sets and relations.

Another important property the coderiving transformation satisfies is its relation with the deriving transformation.

Theorem 16. The deriving and coderiving transformations satisfy the following equality:

$$
\mathrm{d}_{A} \mathrm{~d}_{A}^{\circ}=\mathrm{W}_{A}+1_{! A \otimes A}
$$

where $\mathrm{W}$ is the natural transformation with components $\mathrm{W}_{A}=\left(\mathrm{d}_{A}^{\circ} \otimes 1_{A}\right)\left(1_{! A} \otimes \sigma\right)\left(\mathrm{d}_{A} \otimes 1_{A}\right)$. 
The notation W was introduced by Ehrhard's in [11] where a proof can be found. In the graphical calculus, the above identity is expressed as follows:

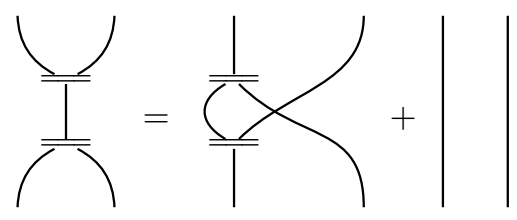

In a differential category there are two important natural transformations $\mathrm{K}$ and $\mathrm{J}$ defined by $\mathrm{K}_{A}:=\mathrm{d}_{A}^{\circ} \mathrm{d}_{A}+! 0: ! A \rightarrow ! A$ and $\mathrm{J}_{A}:=\mathrm{d}_{A}^{\circ} \mathrm{d}_{A}+1_{! A}: ! A \rightarrow ! A$, written in the graphical calculus as:

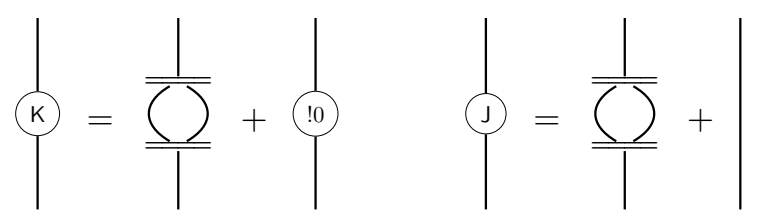

$\mathrm{K}$ and $\mathrm{J}$ satisfy a long list of very similar properties which describe their interaction with the differential structure. We give some of the more important ones in the following theorem:

- Theorem 17. $\mathrm{K}$ and $\mathrm{J}$ satisfy the following properties:

[K.1] $\mathrm{K} !(0)=!(0)=!(0) \mathrm{K}$;

[K.2] $\mathrm{K} e=e$;

[K.3] $\mathrm{K} \varepsilon=\varepsilon$;

[K.4] $\mathrm{K} \Delta=\Delta\left(\left(\mathrm{dd}^{\circ}\right) \otimes 1\right)+\Delta\left(1 \otimes\left(\mathrm{dd}^{\circ}\right)\right)+\Delta(!(0) \otimes !(0))$;

[K.5] $\mathrm{K} \delta=\delta \mathrm{d}^{\circ}\left(1 \otimes\left(\mathrm{dd}^{\circ}\right)\right) \mathrm{d}+\delta !(!(0))$;

[K.6] $(\mathrm{K} \otimes 1) \mathrm{W}=\mathrm{W}(\mathrm{K} \otimes 1)$;

[K.7] $(\mathrm{K} \otimes 1) \mathrm{dd}^{\circ}=\mathrm{dd}^{\circ}(\mathrm{K} \otimes 1)$.

[J.1] $\mathrm{J} !(0)=!(0)=!(0) \mathrm{J}$;

[J.2] $\mathrm{J} e=e$;

[J.3] $\mathrm{J} \varepsilon=2 \cdot \varepsilon$;

[J.4] $\mathrm{J} \Delta=\Delta(\mathrm{J} \otimes 1)+\Delta\left(1 \otimes\left(\mathrm{dd}^{\circ}\right)\right)=\Delta\left(\left(\mathrm{dd}^{\circ}\right) \otimes 1\right)+\Delta(1 \otimes \mathrm{J}) ;$

[J.5] $\mathrm{J} \delta=\delta \mathrm{d}^{\circ}\left(1 \otimes\left(\mathrm{dd}^{\circ}\right)\right) \mathrm{d}+\delta$;

[J.6] $(\mathrm{J} \otimes 1) \mathrm{d}=\mathrm{dK}$;

[J.7] $\mathrm{d}^{\circ}(\mathrm{J} \otimes 1)=\mathrm{Kd}^{\circ}$;

[J.8] $(\mathrm{J} \otimes 1) \mathrm{W}=\mathrm{W}(\mathrm{J} \otimes 1)$;

[J.9] $(\mathrm{J} \otimes 1) \mathrm{dd}^{\circ}=\mathrm{dd}^{\circ}(\mathrm{J} \otimes 1)$.

Recall that Ehrhard's original idea was to obtain integration by requiring that $\mathrm{J}$ be a natural isomorphism. However, Ehrhard's integral transformation, using only that $\mathrm{J}$ is invertible, appears to fail the Rota-Baxter rule [s.2]. This is why we have strengthened Ehrhard's approach by requiring instead that $\mathrm{K}$ be a natural isomorphism. We observe:

- Theorem 18. For a differential category, $\mathrm{K}$ is a natural isomorphism if and only if $\mathrm{J}$ is a natural isomorphism and the deriving transformation is Taylor. 
Proof. We give the definitions of $\mathrm{K}^{-1}$ and $\mathrm{J}^{-1}$ :

(i) $\Rightarrow$ (ii) If $\mathrm{K}$ is a natural isomorphism, then: $\mathrm{J}_{A}^{-1}:=\delta_{A} \mathrm{~K}_{! A}^{-1} \mathrm{~d}_{! A}^{\circ}\left(!\left(\varepsilon_{A}\right) \otimes e_{A}\right) \rho_{!}$, written in the graphical calculus as:

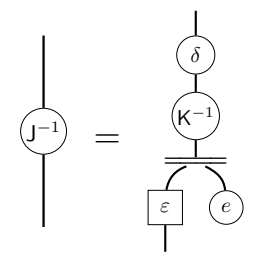

(ii) $\Rightarrow$ (i) If $\mathrm{J}$ is a natural isomorphism and the deriving transformation is Taylor, then $\mathrm{K}_{A}^{-1}:=\mathrm{d}_{A}^{\circ}\left(\mathrm{J}_{A}^{-1} \otimes 1_{A}\right)\left(\mathrm{J}_{A}^{-1} \otimes 1_{A}\right) \mathrm{d}_{A}+! 0$, where $0: A \rightarrow A$, and written in the graphical calculus as:

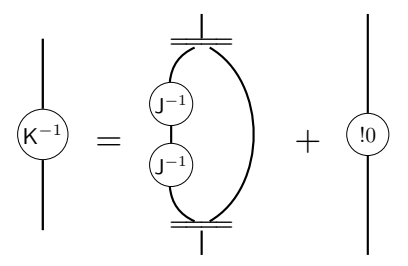

Definition 19. A differential category has antiderivatives if $\mathrm{K}$ is a natural isomorphism.

Equivalently, of course, a differential category has antiderivatives if $\mathrm{J}$ is a natural isomorphism and the deriving transformation is Taylor. While our definition of antiderivatives differs only slightly from Ehrhard's, [11], our definition does imply Ehrhard's definition and, at the same time, secures the property of being an integral category for which, as far as we can see, inverting $\mathrm{J}$ is insufficient.

- Theorem 20. In a differential category with antiderivatives, $\mathrm{K}^{-1}$ and $\mathrm{J}^{-1}$ satisfy the following properties:

$\left[\mathrm{K}^{-1} .1\right] \mathrm{K}^{-1} !(0)=!(0)=!(0) \mathrm{K}^{-1}$;

$\left[\mathrm{K}^{-1} .2\right] \mathrm{K}^{-1} e=e$;

$\left[\mathrm{K}^{-1} .3\right] \mathrm{K}^{-1} \varepsilon=\varepsilon$;

$\left[\mathrm{K}^{-1} .4\right] \Delta\left(\mathrm{K}^{-1} \otimes \mathrm{K}^{-1}\right)+\Delta\left(\mathrm{K}^{-1} \otimes !(0)\right)+\Delta\left(!(0) \otimes \mathrm{K}^{-1}\right)=\mathrm{K}^{-1} \Delta\left(\mathrm{K}^{-1} \otimes 1\right)+\mathrm{K}^{-1} \Delta(1 \otimes$

$\left.\mathrm{K}^{-1}\right)+\Delta(!(0) \otimes !(0))$

$\left[\mathrm{K}^{-1} .5\right]\left(\mathrm{K}^{-1} \otimes 1\right) \mathrm{W}=\mathrm{W}\left(\mathrm{K}^{-1} \otimes 1\right)$;

$\left[\mathrm{K}^{-1} .6\right]\left(\mathrm{K}^{-1} \otimes 1\right) \mathrm{dd}^{\circ}=\mathrm{dd}^{\circ}\left(\mathrm{K}^{-1} \otimes 1\right)$;

$\left[\mathrm{J}^{-1} .1\right] \mathrm{J}^{-1} !(0)=!(0)=!(0) \mathrm{J}^{-1}$;

[ $\left.\mathrm{J}^{-1} .2\right] \mathrm{J}^{-1} e=e$;

[J-1.3] $2 \cdot \mathrm{J}^{-1} \varepsilon=\varepsilon$;

$\left[\mathrm{J}^{-1} .4\right]\left(\mathrm{J}^{-1} \otimes 1\right) \mathrm{d}=\mathrm{dK}^{-1}$;

$\left[\mathrm{J}^{-1} .5\right] \mathrm{d}^{\circ}\left(\mathrm{J}^{-1} \otimes 1\right)=\mathrm{K}^{-1} \mathrm{~d}^{\circ}$;

$\left[\mathrm{J}^{-1} .6\right]\left(\mathrm{J}^{-1} \otimes 1\right) \mathrm{W}=\mathrm{W}\left(\mathrm{J}^{-1} \otimes 1\right)$;

$\left[\mathrm{J}^{-1} .7\right]\left(\mathrm{J}^{-1} \otimes 1\right) \mathrm{dd}^{\circ}=\mathrm{dd}^{\circ}\left(\mathrm{J}^{-1} \otimes 1\right)$;

In particular, $\left[\mathbf{J}^{-1} . \mathbf{5}\right]$ will imply that the integral transformation constructed using either $\mathrm{K}^{-1}$ or $\mathrm{J}^{-1}$ are equal to one another. Finally, with these properties of $\mathrm{K}^{-1}$ and $\mathrm{J}^{-1}$, we obtain the main result of this section, namely that a differential category with antiderivatives is a calculus category: 
- Theorem 21. A differential category with antiderivatives is a calculus category with the integral transformation defined by $\mathrm{s}_{A}:=\mathrm{K}_{A}^{-1} \mathrm{~d}_{A}^{\circ}=\mathrm{d}_{A}^{\circ}\left(\mathrm{J}_{A}^{-1} \otimes 1_{A}\right)$, expressed in the graphical calculus as:<smiles>CCC(C(C)CC(C)C)C(CC)(CC)CC</smiles>

Proof. To prove the integral transformation axioms and the second fundamental theorem we use the $\mathrm{K}^{-1}$ form of the integrating transformation. While to prove the Poincaré condition we use Ehrhard's $\mathrm{J}^{-1}$. We will use the graphical calculus to help us. We first show that our integral transformation satisfies $[\mathbf{s . 1}]$ to $[\mathbf{s . 3}]$.

[s.1]: Here we use $[\mathbf{c d . 1}]$ and $\left[\mathbf{K}^{-1} . \mathbf{2}\right]$ :

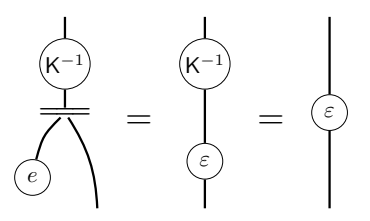

[s.2]: Here we use $\left[\mathbf{K}^{-1.4}\right],[\mathbf{c d . 3}],[\mathbf{c d . 4}]$ and naturality of the coderiving transformation:

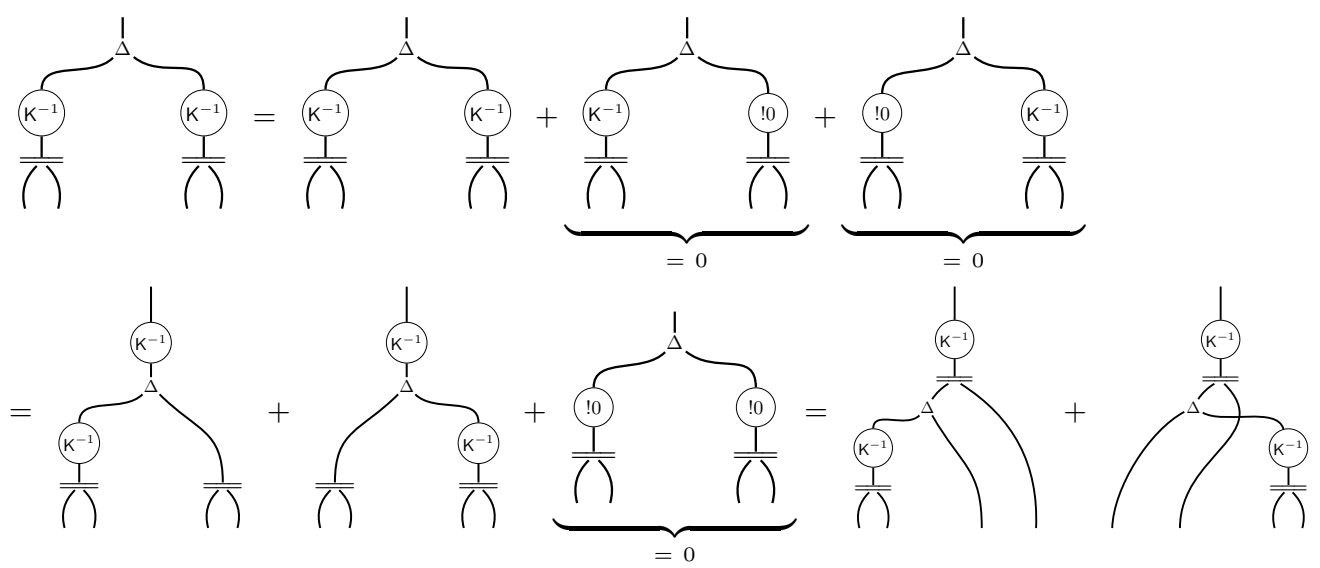

$[\mathbf{s . 3}]:$ Here we use $\left[\mathbf{J}^{-1} . \mathbf{5}\right]$ and $[\mathbf{c d . 7}]$ :

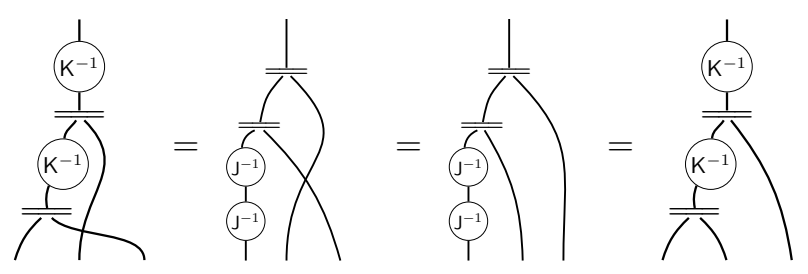

Next we show the second fundamental theorem of calculus. Here we use $\left[\mathbf{K}^{-1} \mathbf{. 1}\right]$ :

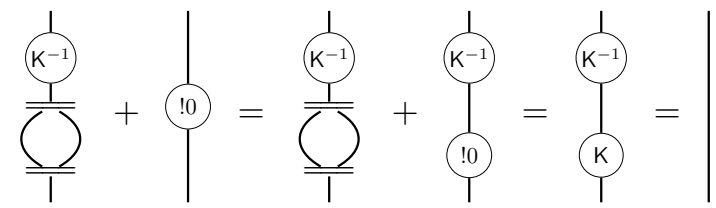


Finally we prove the Poincaré Condition. Let $f: C \otimes ! A \otimes A \rightarrow B$ satisfy the Poincaré pre-condition, that is, $(1 \otimes 1 \otimes \sigma)(1 \otimes \mathrm{d} \otimes 1) f=(1 \otimes \mathrm{d} \otimes 1) f$. First notice that by Theorem 16 and the Poincaré pre-condition, $f$ satisfies the following identity:

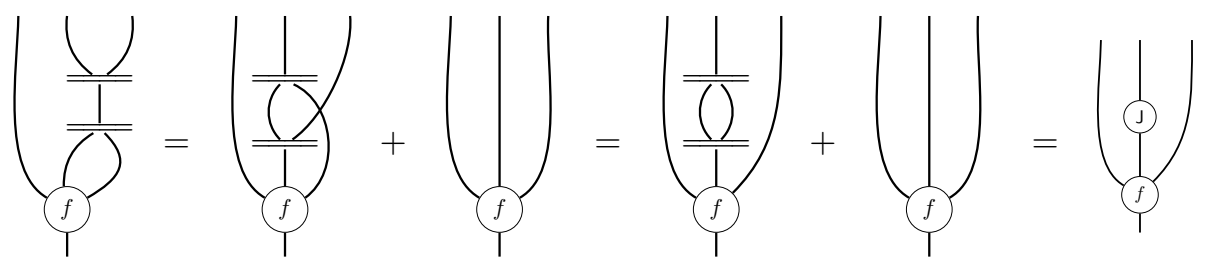

Then using $\left[\mathbf{J}^{-1} \cdot \mathbf{4}\right]$ and the above identity we get the following equality:

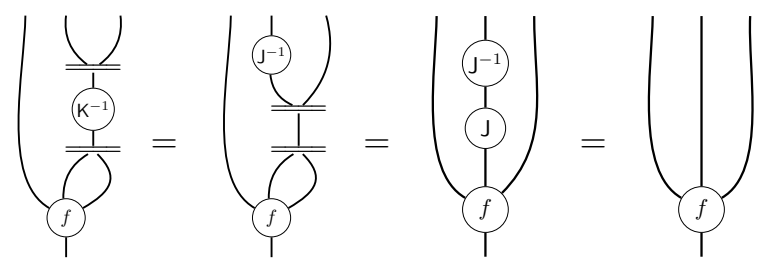

Which completes the proof that antiderivatives give a calculus category.

The converse of Theorem 21 is true if the coalgebra is monoidal (in the sense explained at the end of Section 3 when discussing Fubini's theorem) and the integral transformation is compatible with monoidal strength, that is, a calculus category with a monoidal coalgebra modality and a monoidal integral transformation is a differential category with antiderivatives. More details and a proof of this will be given in a subsequent paper.

We are now are in a position to give two examples of differential categories which have antiderivatives, and therefore, two examples of calculus categories:

- Example 22. The category of sets and relations, REL, is a differential category [7] with antiderivatives. The symmetric monoidal strucure is given by the Cartesian product of sets while the additive structure is given by the union of sets. The coalgebra modality is given by the finite bag/multiset comonad (see [7] for more details), where for a set $X, ! X$ is the set of bags/multisets of $X$. The deriving transformation $\mathrm{d}_{X}: ! X \times X \rightarrow ! X$ is the relation which adds an extra element to the bag:

$$
\mathrm{d}_{X}=\{((B, x), B \cup x) \mid x \in X, B \in ! X\}
$$

The additive idempotency of REL makes both $\mathrm{K}$ and $\mathrm{J}$ the identity and thus trivially isomorphisms. Therefore, the integral transformation is the coderiving transformation $\mathrm{d}_{X}^{\circ}: ! X \rightarrow ! X \times X$, which is the relation which removes an elements from the bag:

$$
\mathrm{d}_{X}^{\circ}=\{(B,(B-\{x\}, x)) \mid x \in X, B \in ! X\}
$$

- Example 23. The category of vector spaces over a field $\mathbb{K}$ of characteristic of $0, V C_{\mathbb{K}}$, is a co-differential category [7] with antiderivatives, so that, $\mathrm{VEC}_{\mathbb{K}}^{\mathrm{op}}$ is a calculus category. While having a field of characteristic zero is not required to obtain differential structure, it is required for antiderivatives. The additive symmetric monoidal structure is given by the standard tensor product and additive enrichment of vector spaces. The algebra modality is given by the free symmetric algebra monad where for a vector space $V, ! V$ is the free commutative algebra over $V$ (see [17] for more details). Equivalently, if $X=\left\{x_{1}, x_{2}, \ldots\right\}$ is 
a basis of $V$, then $! V$ is isomorphic to the polynomial ring over $X: ! V \cong \mathbb{K}[X][17]$. Then the deriving transformation $\mathrm{d}_{V}: ! V \rightarrow ! V \otimes V$ (recall $\mathrm{VEC}_{\mathbb{K}}^{\text {op }}$ is the calculus category) on monomials is given by the sum of partial derivatives of the monomial:

$$
\mathrm{d}_{V}\left(x_{1}^{r_{1}} \ldots x_{n}^{r_{n}}\right)=\sum_{i=1}^{n}\left(x_{1}^{r_{1}} \ldots x_{i}^{r_{i}-1} \ldots x^{r_{n}}\right) \otimes x_{i}
$$

On monomials, $\mathrm{K}$ multiplies the non-constant monomials by their degree and multiplies the constants by one, while $\mathrm{J}$ multiplies monomials by their degree plus one. As the rationals are embedded in our field, both are isomorphisms, and the resulting integral transformation $\mathrm{s}_{V}: ! V \otimes V \rightarrow ! V$ is defined on monomials by:

$$
\mathrm{s}_{V}\left(\left(x_{1}^{r_{1}} \ldots x_{n}^{r_{n}}\right) \otimes x_{i}\right)=\frac{1}{1+\sum_{j=1}^{n} r_{j}} x_{1}^{r_{1}} \ldots x_{i}^{r_{i}+1} \ldots x^{r_{n}}
$$

At first glance this may seem bizarre. One might expect the integrating transformation to integrate a monomial with respect to the variable $x_{i}$ and thus only multiply by $\frac{1}{1+r_{i}}$. However, this classical idea of integration fails the Rota-Baxter rule [s.2] for any vector space of dimension greater than one.

\section{Conclusion and Future Work}

The theory of differential categories was developed in stages: (tensor) differential categories [7], cartesian differential categories [3], differential restriction categories [10], and tangent categories [9]. The development of integral categories, being very closely related, has parallel stages. Here we have briefly introduced the first stage of this development: tensor integral categories. The next stage, Cartesian integral categories, is actually well in hand. The coKleisli category of an integral category is a Cartesian integral category. Furthermore, Cartesian integral categories have a term logic which has a more "classic" feel: we borrowed parts of this term logic to help motivate this paper. The study of integration in restriction categories and tangent categories is, by comparison, in its earliest stages.

Acknowledgements. The authors would like to thank Rick Blute for drawing both authors' attention to Rota-Baxter algebras. Integral categories simply would not have developed so rapidly without this basic inspiration. Jonathan Gallagher reminded us of Ehrhard's work at exactly the right moment, while Kristine Bauer provided continual constructive criticism during the evolution of our thoughts.

\section{References}

1 Glen Baxter et al. An analytic problem whose solution follows from a simple algebraic identity. Pacific J. Math, 10(3):731-742, 1960.

2 R. Blute, J.R. B. Cockett, and R. A. G. Seely. Cartesian differential storage categories. Theory and Applications of Categories, 30(18):620-686, 2015.

3 R.F. Blute, J. Robin B. Cockett, and R. A. G. Seely. Cartesian differential categories. Theory and Applications of Categories, 22(23):622-672, 2009.

4 Richard Blute, J.R. B. Cockett, Timothy Porter, and R. A. G. Seely. Kähler categories. Cahiers de Topologie et Géométrie Différentielle Catégoriques, 52(4):253-268, 2011.

5 Richard Blute, Thomas Ehrhard, and Christine Tasson. A convenient differential category. arXiv preprint arXiv:1006.3140, 2010. 
6 Richard Blute, Rory B. B. Lucyshyn-Wright, and Keith O'Neill. Derivations in codifferential categories. arXiv preprint arXiv:1505.00220, 2015.

7 Richard F. Blute, J. Robin B. Cockett, and Robert A. G. Seely. Differential categories. Mathematical structures in computer science, 16(06):1049-1083, 2006.

8 Raoul Bott and Loring W. Tu. Differential forms in algebraic topology, volume 82. Springer Science \& Business Media, 2013.

9 J. Robin B. Cockett and Geoff S. H. Cruttwell. Differential Structure, Tangent Structure, and SDG. Applied Categorical Structures, 22(2):331-417, 2014.

10 J.R. B. Cockett, G. S. H. Cruttwell, and J. D. Gallagher. Differential restriction categories. Theory and Applications of Categories, 25(21):537-613, 2011.

11 Thomas Ehrhard. An introduction to differential linear logic: proof-nets, models and antiderivatives. Mathematical Structures in Computer Science, pages 1-66, 2017.

12 Thomas Ehrhard and Laurent Regnier. The differential lambda-calculus. Theoretical Computer Science, 309(1):1-41, 2003.

13 Thomas Ehrhard and Laurent Regnier. Differential interaction nets. Theoretical Computer Science, 364(2):166-195, 2006.

14 Marcelo P. Fiore. Differential structure in models of multiplicative biadditive intuitionistic linear logic. In International Conference on Typed Lambda Calculi and Applications, pages 163-177. Springer, 2007.

15 Li Guo. An introduction to Rota-Baxter algebra, volume 2. International Press Somerville, 2012.

16 André Joyal and Ross Street. The geometry of tensor calculus, I. Advances in Mathematics, 88(1):55-112, 1991.

17 Serge Lang. Algebra revised third edition. Graduate Texts in Mathematics, 1(211):ALLALL, 2002.

18 J.-S. P. Lemay. Integral Categories and Calculus Categories. University of Calgary, 2017.

19 Saunders Mac Lane. Categories for the working mathematician, volume 5. Springer Science \& Business Media, 2013.

20 Gian-Carlo Rota. Baxter algebras and combinatorial identities. I. Bulletin of the American Mathematical Society, 75(2):325-329, 1969.

21 Peter Selinger. A survey of graphical languages for monoidal categories. In New structures for physics, pages 289-355. Springer, 2010.

22 Charles A. Weibel. An introduction to homological algebra. Cambridge university press, 1995. 